\title{
A PROPOS DE LA CHUTE DU ROYAUME DE ÇRIVIJAYA
}

PAR

G. CQEDÈS.

M. N. J. Krom vient de publier dans les ,Mededeelingen der Koninklijke Akademie van Wetenschappen, Afdeeling Letterkunde” (Deel 62, Serie B, No. 5, Amsterdam 1926, pp. 149-171) un mémoire sur la chute de Çrīvijaya (De ondergang van Çrīwijaya) qui est un remarquable essai de synthèse historique. La thèse du savant professeur à l'Université de Leiden peut se résumer ainsi :

La chute de Çrivijaya ne date pas de 1376 - 1377, comme on a cru autrefois pouvoir le déduire de l'Histoire des Ming, qui place à cette date la conquête du San-fo-ts'i par les Javanais. D'autre part, le Malāyu, dont le nom, oublié depuis la conquête de ce pays par Çrīvijaya à la fin du VIIme siècle, reparait vers 1280, n'est pas „le royaume de Çrivijaya sous un autre nom”. Durant le dernier quart du XIIIme siècle, la suzeraineté sur les différents royaumes de Sumatra et de la Péninsule Malaise a passé de Çrivijaya au Malāyu. La cause initiale de la décadence de Çrīvijaya est l'expédition malheureuse du roi Candrabhānu contre Ceylan en 1251. „A la suite de cette catastrophe, dit M. Krom, Çrivijaya n'a plus pu résister aux invasions combinées de ses ennemis dans le Nord et dans le Sud: les Thai de Sukhodaya s'emparèrent d'une partie de la Péninsule Malaise, le roi Krtanagara de Java dirigea une expédition contre Sumatra, où l'état de Malāyu, vivant jusqu'alors sous la domination de Çrīvijaya reconquit son indépendance grâce à la protection javanaise... La chute véritable de Çrivijaya est celle de \pm 1280 ".

Non seulement je crois que M. Krom a raison de reculer de plus d'un siècle la chute de Çrivijaya, mais je pense même pouvoir montrer que la décadence du grand royaume sumatranais remonte encore plus haut. Je n'ai pas à proprement parler de pièces nouvelles à 
verser au débat, mais un nouvel examen de certains documents publiés pour la première fois en 1918 en appendice à mon étude sur „le Royaume de Çrīvijaya” (B. E. F. E.-O., XVIII, vI) m'a amené à compléter et à corriger l'interprétation que j'en avais donnée. Après l'accueil flatteur que mes confrères hollandais, et particulièrement $\mathrm{M}$. Krom, ont réservé à mon mémoire, je me considère comme un peu responsable des théories qu'ils ont échafaudées sur la mienne, et je leur dois de les tenir au courant des modifications ou des améliorations que je crois devoir $\mathrm{y}$ apporter.

L'expédition de Candrabhānu à Ceylan est connue par deux passages du Mahāvamsa (LXXXIII, 36-48 et LXXXVIII, 62-75) signalés pour la première fois par Kern (Bijdr., XLVI, 1896) et utilisés par Ferrand (L'empire sumatranais de Çrīvijaya, p. 228). On me permettra de reproduire ici deux fragments du texte pāli qui contiennent certaines données dont on n'a pas encore tiré tout le parti possible.

athekādasame vasse sampatte tassa rājino tadā jāvakarājeko Candabhānūti vissuto vayam pi Sogatā eva iti māyamı vidhāya so saddhim jāvakasenāya Kakkhalāya samotari sabbe te Jāvakā yodhā sabbatitthāvagāhino visadiddhehi bānehi ghorasappanibhehi ye ditțhedițthe jane duțthā bādhamānā nirantaram kodhā sandhāvamānā tu sabbạn Laìkam vināsayum

(LXXXIII, 36-39).

„Ce fut dans la onzième année du règne de ce roi (Parākramabāhu II), qu'un certain roi des Jāvaka nommé Candrabhānu débarqua à Kakkhala avec une armée de Jāvaka, après avoir traitreusement déclaré: „Nous aussi nous sommes des Bouddhistes”. Les guerriers Jāvaka, qui étaient tous armés de flèches empoisonnées comparables à d'horribles serpents, se rendirent maitres de tous les gués de rivières, et tourmentant cruellement tous ceux qu' ils rencontraient, ils ruinèrent Lañkā qu'ils avaient parcourue comme des furieux en tous sens".

La suite du texte relate comment le régent Vīrabāhu, dépêché par le roi son oncle, repoussa victorieusement les Jāvaka et en libéra le territoire.

Et plus loin: 
tasmim kāle mahāyuddham katvā pubbe palāpito

Candabhānunarindo so Pandu-Colädirațthato

mahā-Dāmilayodhe ca rāsikatvā mahabbalo

saddhị Jävakasenāya Mahātițtham samotari

atho Padi-Kurundādiratthavāāī ca Sīhale

vasikatvāna rājā so gantvāna Subhapabbatam

khandhāvārạ̣ nibandhitvā gaṇhissāmīti Sīhalam

na harissāmi te tasmā Munino dantadhātuyā

saheva pattadhātuñ ca rajjañ ca mama dehi tam

no ce yuddham karohīti vatvā dūte ca pesayi

tadē Vijayabāhu pi Vīrabāhumah̄̄patim

pakkosetvābhimantetvā sajjāpetvā mahābalam

(LXXXVIII, 62-66).

„Ce fut à cette époque que le roi Candrabhānu qui auparavant avait été repoussé après une grande bataille, rassembla dans les royaume Pāṇya et Cola une grande armée de guerriers tamils et débarqua à Mahātițtha avec une bande de Jāvaka. Après avoir soumis les Singhalais habitant les districts de Padi, Kurunda et autres, il se dirigea vers le mont Subha où il installa son quartier général. Il envoya alors (au roi de Ceylan) des ambassadeurs avec ce message: ,Je vais m'emparer de Ceylan; aussi ne vous prendrai-je pas (de force) la dent-relique du Muni. Donnez-la moi, avec le bolrelique et la royauté; sinon, préparez-vous au combat". Alors Vijayabāhu (fils de Parākramabāhu, à qui ce dernier avait confié le pouvoir) fit appeler le régent Vĩrabāhu, tint conseil avec lui et fit équiper une grande armée."

Le résultat du combat est une nouvelle défaite de Candrabhānu.

Telles sont les données du Mahāvamsa.

Kern, écrivant à une époque où l'histoire de l'archipel n'avait pas encore fait les progrès auxquels on a assisté durant ces dernières années, avait tout naturellement traduit Jāvaka par ,Javanais”. Dans sa version du Mahāvamsa, L. C. Wijesinha, se basant probablement sur le sens moderne du mot, rend Jävaka par „Malais”. Ferrand (L'empire sumatranais de Çrīvijaya, p. 115, 172), ayant retrouvé dans jāvaka l'origine de la transcription $Z \bar{a} b a d j$ (anciennement $Z \bar{a} b a g$ ) des géographes arabes, et ayant identifié à juste titre le pays de Zābag ou empire du Mahārāja avec Çrīvijaya, a été amené à faire de Candrabhānu un roi de Çrīvijaya. C'était, dans 
l'état des connaissances, la seule solution qui se présentât à l'esprit, et personne ne saurait reprocher à M. Krom de l'avoir adoptée et de parler d'une ,campagne de Çrivijaya à Ceylan”.

C'est moi au contraire qui dois m'accuser publiquement de n'avoir pas su reconnaitre le nom de Candrabhānu dans une inscription de Jaiya que j'ai publiée en 1918 en appendice au „Royaume de Çrīvijaya” (p. 32), et d'où il ressort que Candrabhānu n'est pas un roi de Çrīvijaya.

Il s'agit de l'inscription en sanskrit incorrect datée kaliyuga 4332, soit 1230 A.D. Le nom de Candrabhānu y apparait deux fois, une première fois (1.3) dans une sorte de calembour: samrüpena $h i$ candrabhānumadanạ̣ çrīdharmmarājā, ,,(le roi) Çrīdharmarāja qui par son (bel) aspect était Madana (le dieu de l'Amour) ayant l'éclat (bhānu) de la lune (candra)”, ou bien ,était (à la fois) la lune, le soleil et Madana", - une seconde fois (1.7): Candrabhānūpādhiçrīdharmmarājāa, ,(le roi) Çrīdharmarāja ayant nom Candrabhānu”. Il est donc hors de doute que le roi qui fit graver l'inscription de Jaiya en 1230 se nommait Candrabhānu.

Fort bien, dira-t-on, mais qu'est-ce qui empêche que ce soit un roi de Çrivijaya faisant une fondation pieuse dans un des états tributaires de son empire? N'a-t-on pas vu en 775 un autre roi de Çrīvijaya construire un sanctuaire bouddhique dans la même région, et l'inscription du Buddha de Grahi n'a-t-elle pas pour auteur un roi du Malāyu?

A regarder de plus près cette inscription de Jaiya, il semble bien difficile de voir dans Candrabhānu autre chose qu'un souverain local. S'il était réellement le Mahārāja de Çrīijaya, il est presque certain que, suivant l'exemple de son prédecesseur qui fit la fondation de $775^{1}$ ), il aurait mentionné son titre de "Seigneur de Çrivijaya" (Çrīijayesvara) au lieu de s'intituler simplement „Seigneur de Tāmbralinga" (Tāmbralingeçvara). D’autre part, le titre de Çrīdharmarāja, qui ne figure à ma connaissance dans aucun document émanant de la cour de Palembang, semble bien être un titre particulier et héréditaire des rois de Ligor: lorsque, vers le milieu du

1) C'est l'inscription dite de Vieng Sra, mais qui en réalité vient de Ligor. Je l'avais appelée en 1918 ,stèle de Vieng Sra” sur la foi d'un renseignement dû à $\mathrm{M}$. de Lajonquière et reproduit par M. Finot (Bull. Comm. arch. de l'Indochine, 1910, pp. 149, 152). D'une enquète à laquelle je me suis livré récemment sur les lieux, il résulte qu'il n'y a jamais eu d'inscription à Vieng Sra, d'où proviennent seulement des statues brâhmaniques, mais que la stèle de 775 vient du Vat Semā Müang de Ligor. 
XIIIme siècle, les Thai entrèrent en contact avec cette région, ils donnèrent à la capitale du Tāmbralinga le nom, qu'elle porte encore aujourd'hui, de Nagara Çrīdharmarāja. Enfin, les familles auxquelles se rattache Candrabhānu, le Padmavamça (1.2) et le Pañcāṇavamçça (quel que soit le sens de cette expression énigmatique), sont complètement différentes du Çailendravamça dont se réclamaient les rois de Çrīvijaya. Tous ces faits concourent à donner l'impression que Candrabhānu était, non le Mahārāja de Çrīijiaya, mais le roi, plus ou moins indépendant, d'un état de la Péninsule Malaise.

Est-il légitime de l'identifier avec Candrabhānu, roi des Jāvaka, qui selon le Mahāvamsa vint à deux reprises attaquer Ceylan sous le règne de Parākramabāhu?

Le terme de Jāvaka, employé par le Mahāvamsa, tout en correspondant phonétiquement à Zābag comme le dit Ferrand, n'est pas forcément l'équivalent géographique de Zäbag, en tant que ce nom désigne chez les Arabes l'Empire du Mahārāja de Çrīijijaya. Ce peut être simplement un nom ethnique désignant les Indonésiens, et ayant à peu près le même sens que le mot cambodgien moderne Javā (prononcé chvéa) qui s'applique aussi bien aux Malais de la Péninsule qu'à ceux des îles et aux Javanais. Rien de moins précis que ces noms par lesquels les peuples orientaux se désignent les uns les autres. Dans le cas présent, l'appellation de Jāvaka appliquée par le Mahāvamsa aux habitants du Tāmbralinga, c'est à dire de la région de Ligor, est justifiée, d'une part parce que cet état avait été le vassal de Çrīvijaya et dépendait peut-être encore de Sumatra, et d'autre part parce que la population du Tāmbralinga lui-même, avant l'arrivée des Thai, ne devait pas être foncièrement différente de celle de Sumatra, le pays Jāvaka par excellence.

Le Candrabhānu de l'inscription de Jaiya était un roi bouddhiste. Il ,procurait une félicité extrême à la religion du Buddha" (1. 1) et la fondation qui faisait l'objet de ce texte était une fondation bouddhique. Or, on a vu que, lors de sa première campagne à Ceylan, le Candrabhānu du Mahāvamsa commence par déclarer que „eux aussi sont des bouddhistes", et que, lors de sa seconde expédition, il réclame la dent et le bol du Buddha.

Est-il possible enfin qu'un roi qui régnait à Ligor en 1230 ait attaqué Ceylan aux dates citées par le Mahāvamsa? En réalité, le Mahāvamsa ne donne aucun millésime. Il dit seulement que la première attaque eut lieu ,la onzième année du règne de Parākrama- 
bāhu". Quant à la seconde, elle se place à une époque où ce roi "après avoir joui de la royauté durant de nombreuses années" (LXXXVII, 16) avait déjà remis à son fils Vijayabāhu la direction des affaires. Parākramabāhu ayant régné 35 ans, on peut en gros placer cette seconde invasion de Candrabhānu pendant le dernier tiers de son règne. Il s'agit naturellement de Parākramabāhu II. Kern qui s'était servi, à l'époque de sa première publication dans les Bijdragen de 1896 (Deel XLVI), de l'histoire de Ceylan par Knighton (1845) avait cru qu'il s'agissait de Parākramabāhu III. Rouffaer, dans une note de son „Was Malaka emporium ...” (Bijdr. LXXVII, 1921 , p. 83, note 1) rectifia cette indication manifestement erronée, rapporta correctement les invasions de Candrabhānu au règne de Parākramabāhu II, et, adoptant la chronologie de la traduction de Wijesinha qui fait régner ce roi de 1240 à 1275 , plaça ces deux invasions en 1251 et circa 1255 . Cette chronologie a été acceptée par Ferrand (L'empire sumatranais de Çrivijaya, pp. 172-173) et par M. Krom (p. 8). Mais, d'une communication personnelle que je dois à l'obligeance de $\mathrm{M}$. Jouveau-Dubreuil, il résulte que pour la fin du XIIme siècle au moins, les dates de Wijesinha sont trop basses d'une quinzaine d'années.

On sait que le roi Parākramabāhu I prit parti dans la guerre dite „de succession Pāṇ̣̂a" et envoya deux de ses généraux avec une puissante armée en pays tamoul. „Or, m'écrit M. Jouveau-Dubreuil, l'inscription No. 20 du Rapport sur l'épigraphie de la Présidence de Madras pour 1899 prouve que l'intervention de Ceylan, ainsi que celle des Cola, dans la guerre Pānḍya avait déjà eu lieu dans la cinquième année du règne de Rãjādhirāja II, qui commença à régner en 1163. La guerre de succession Pāṇ̣ya commença donc entre 1163 et 1168 , et elle se termina avant 1182 , car d'après une autre inscription (Rapport pour 1917-1918, p. 151), les Cola étaient vainqueurs à cette date. Or le Mahāvamsa place cette guerre entre la seizième année et la fin du règne de Parākramabāhu I, c'est à dire, d'après la chronologie de Wijesinha, entre 1180 et 1197 . On peut donc dire que pour la fin du XIIme siècle, les dates de Wijesinha sont trop basses d'environ 15 ans".

Si l'on applique provisoirement cette correction de 15 ans au règne de Parākramabāhu II, il s'ensuit que ce roi régna de 1225 à 1260 . La première invasion de Candrabhānu, qui eut lieu dans la onzième année du règne de Parākramabāhu II, daterait de 1236 (6 ans après l'inscription de Jaiya), et la seconde, qui se place dans le dernier tiers 


\section{INSCRIPTION DE GRAHI.}

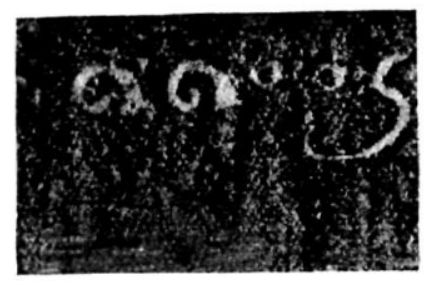

LA DATE.

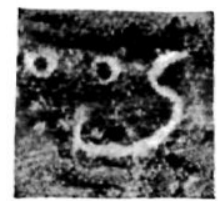

LES DEUX DERNIERS CHIFFRES. 
de son règne, aurait eu lieu entre 1250 et 1260 . Or c'est exactement à cette époque, en 1256, que l'ouvrage historique pāli nommé Jinakālamālinī place une expédition, pacifique il est vrai, envoyée à Ceylan par le roi Siridhammarāja de Siridhammanagara, et par Rocarāja, prince thai de Sukhodaya, pour rapporter la fameuse statue du Brạ Sihing . $\left.{ }^{1}\right)$ Qu'une expédition présentée par le Mahāvamsa comme une invasion hostile soit devenue dans la Jinakālamālinī, composée à Chieng Mai au début du XVIme siècle, une ambassade pacifique, il n'y a là rien d'impossible. La religion semble avoir été intimement mêlée à cette histoire: on a vu que Candrabhānu, qui s'était écrié en débarquant à Ceylan pour la première fois „Nous aussi nous sommes des bouddhistes", réclama la seconde fois la dent et le bol du Buddha. Dès l'origine du bouddhisme, le partage des cendres du Maitre causa la ,guerre des reliques”, et la statue du Brạ Sihing elle-même occasionna au Siam une série de conflits avant de trouver un gite définitif au Musée de Bangkok. Il se peut donc fort bien que les expéditions de Candrabhānu aient eu un but parfaitement pacifique, mais que, par suite de quelque querelle religieuse entre des gens qui ne pratiquaient peut-être pas la même forme de bouddhisme, elle ait dégénéré en une guerre sanglante. Quoi qu'il en soit, il y a certainement quelque rapport entre les faits relatés dans le Mahāvamsa et ceux que fait connaitre la Jinakālamālinī, et c'est, soit dit en passant, une raison de plus pour voir dans Candrabhānu, non un roi de Çrīvijaya, mais le chef d'un état de la Péninsule Malaise.

La correction de 15 ans proposée par M. Jouveau-Dubreuil aux dates de Parākramabāhu I telles que les donne Wijesinha, semble s'appliquer aussi à celles de Parākramabāhu II. Les deux expéditions de Candrabhānu ont dû avoir lieu respectivement en 1236 et en 1256.

Il n'y a donc aucune difficulté chronologique à identifier le Candrabhānu du Mahāvamsa avec celui qui régnait en 1230 sur une partie de la Péninsule Malaise. Puisque les deux expéditions à Ceylan ne sont plus imputables à Çrīvijaya; il n'y a aucune raison pour les considérer comme la cause initiale de la décadence de ce pays. Mais cela ne veut pas dire que ces évènements ne nous renseignent pas indirectement sur la situation du royaume sumatranais vers le milieu du XIIIme siècle. Voici comment.

1) Cœdès, Documents sur l'histoire du Laos occidental, B. E. F. E.-O., $\mathrm{XXV}, 1925$, p. $98-99$.

D1. 83. 
Le texte de l'inscription de Jaiya dit simplement que Candrabhānu était roi du Tāmbralinga et portait le titre de Çrīidharmarāja, c'est à dire qu'il régnait sur la région de Ligor. La présence même de cette inscription à Jaiya, où elle a été trouvée in situ, montre que la domination de Candrabhānu s'étendait jusqu'à la région nord-ouest de la baie de Bandon. L'épigraphie indienne va nous permettre d'ajouter qu'il possédait aussi Kạ̣āram.

Je veux parler des inscriptions de Jațāvarman Vīrapāṇḍa utilisées par Ferrand (p. 48-49), et par M. Krom (p. 9). Ce roi qui commença à régner en $1253 / 1254$ se targue, dans une inscription de la dixième année de son règne (1264), d'avoir conquis Ceylan et d'avoir découronné et décapité le roi des Çāvaka (Jāvaka), et, dans une autre inscription de la onzième année (1265), il énumère parmi ses exploits sa victoire sur le roi de Kaḍāram et sur celui de Ceylan. Ferrand et Krom n'ont pas hésité à retrouver dans ces textes un écho des évènements racontés dans le Mahāvamsa. Ferrand suppose que la conquête de Jāvaka par Jaṭāvarman Virapāṇ̣̣a eut lieu avant l'expédition à Ceylan, et que „Candrabhānu était un simple allié du roi Pāndya dans les opérations entreprises contre Ceylan”. Pour M. Krom, ,soit que Jațāvarman ait été d'abord un allié de Candrabhānu (1e Mahāvaṃsa mentionne les Pāṇ̣ya parmi ses auxiliaires), soit qu'il lui ait été réellement hostile, dans tous les cas, il profita manifestement de l'expédition à Ceylan pour rendre le Jāvaka inoffensif".

Si l'on n'avait que les deux inscriptions de Jațāvarman Virapāṇdya, l'identification de Candrabhānu, allié des Pāṇdya lors de sa seconde campagne à Ceylan en 1256, avec le roi des Jāvaka vaincu et peutêtre tué par ces mêmes Pāṇ̣̂ya, garderait un caractère un peu hypothétique. Ces deux inscriptions ne donnent pas en effet de dates précises et permettent seulement d'affirmer que la conquête de Ceylan et la victoire sur les Jāvaka eurent lieu entre 1254 et 1265 . Il existe heureusement un autre document épigraphique qui permet, je crois, de trancher la question.

Jaṭāvarman Vīrapāṇ̣̂ya eut pour contemporain Jaṭāvarman Sundarapāṇ̣ya I qui régna de 1251 à 1264 . Ces deux rois Pāṇdya devaient être alliés, car leurs panégyriques énumèrent à peu près les mêmes campagnes. Or, dans une inscription de la neuvième année de son règne (1259), Jaṭāvarman Sundarapāṇ̣̂ya I est loué comme ,un autre Rāma parce qu'il avait pillé l'île de Ceylan” (Indian Antiq., 
XXI, p. 343). La campagne des Pāṇ̣ya à Ceylan, que les inscriptions de Jațāvarman Virapāṇ̣̂a permettaient de placer entre 1254 et 1265 , se trouve datée par l'inscription de Jațāvarman Sundarapāṇụa I entre 1251 et 1259 . Elle eut donc lieu entre 1254 et 1259. Le synchronisme entre ces dates et celle de 1256 à laquelle on est arrivé précédenment est trop parfait pour laisser la place au doute. Les Pāṇụa qui accompagnèrent ou aidèrent Candrabhānu à Ceylan en 1256 sont bien Jațāvarman Vīrapāṇ̣̂ya et Jaṭāvarman Sundarapāṇḍya I. Pour quelle raison Jațāvarman Vīrapāṇ̣̂ya se retourna-t-il ensuite contre son ancien allié le roi Jãvaka, il est difficile de le démêler au milieu des renseignements contradictoires que donnent l'épigraphie indienne et la chronique singhalaise. Quel fut exactement le résultat de la campagne de 1256 ? La défaite du roi de Ceylan et du roi des Jāvaka, disent les inscriptions des Pāṇ̣ya; - une victoire pour l'armée singhalaise et la défaite des Jāvaka, dit le Mahāvaṃsa. A y regarder de près, ces réponses en apparence contradictoires sont d'accord sur un point: la défaite des Jāvaka. Le Mahāvaṃsa, en effet, ne parle que de la déroute des Jāvaka, sans plus parler de leurs alliés indiens, dont les prétentions à la victoire ne sont peut-être pas absolument injustifiées. C'est en définitive le malheureux Candrabhānu qui aurait payé tous les frais de son entreprise.

J'ai dit plus haut que ces inscriptions des Pāṇ̣ya enseignent que Candrabhānu possédait Kaḍāram. Voici pourquoi :

L'inscription de 1264 mentionne la victoire sur le roi des Jāvaka, mais ne parle pas de Kaḍāram; celle de 1265 mentionne la victoire sur le roi de Kaḍāram, mais ne parle pas des Jāvaka. L'identification du „roi des Jāvaka” avec le ,roi de Kaḍaram” est la première explication qui se présente tout naturellement à l'esprit. C'est du reste celle qu'ont acceptée Ferrand et Krom, pour qui elle ne pouvait faire aucune difficulté, puisque Candrabhānu, roi des Jāvaka, était d'après eux un roi du Zābag, c'est à dire de Çrīvijaya. Kaḍāram désigne les possessions du Mahārāja sur la Péninsule Malaise, et de même que la grande charte de Leiden de 1006 nomme Māravijayottungavarman „,roi de Kațāha (tamoul: Kị̂ārant) et de Çrĩvișaya”, de même les textes arabes et persans ne cessent de répéter que Kala et le $Z \bar{a} b a g$ sont gouvernés par un même roi. Pour moi qui espère avoir démontré que Candrabhānu n'est pas le Mahārāja de Çrīvijaya, mais le roi du Tāmbralinga, il n'y a pas plus de difficulté à l'entendre appeler roi de Kaḍāram. Kaḍāram - qu'il s'agisse de Kedah ou de Kra — se trouvait sur la côte occidentale de la Péninsule Malaise à la hauteur 
du Tāmbralinga, et l'on conçoit d'ailleurs difficilement que Candrabhānu ait pu préparer et effectuer deux expéditions à Ceylan s'il ne possédait pas un port ayant vue sur le golfe du Bengale. Mais, ce qui est très important, ce qui m'a fait dire plus haut que les campagnes de Candrabhānu à Ceylan nous renseignent indirectement sur la situation de Çrīvijaya à cette époque, c'est de voir le titre de „roi de Kaḍāram”, qui s'appliquait au XIme siècle au Mahārāja de Çrīvijaya, servir à désigner au milieu du XIIIme siècle un roi de la Péninsule Malaise.

Krom a bien montré ${ }^{1}$ ) l'importance capitale que présentait pour Palembang la possession d'établissements sur les côtes de la Péninsule Malaise. Si au milieu du XIIIme siècle Çrīvijaya ne possédait plus Kaḍāram, il n'avait plus la complète maitrise des détroits, et sa décadence avait déjà commencé.

Le Tāmbralinga et les autres petits royaumes de la Péninsule s'étaient-ils simplement rendus indépendants? Le Malāyu était-il déjà entré en scène pour recueillir la succession de son voisin?

Pour en décider, je ne vois guère qu'un document; c'est l'inscription du Buddha de Grahi, dont malheureusement la date est restée jusqu'ici inintelligible.

On sait que cette inscription, que j'ai publiée en 1918 en appendice au „Royaume de Çrīvijaya”, a été trouvée à Jaiya, et qu'elle émane d'un roi nommé Mahārāja çrīmat Trailokyarājamaulibhūṣanavarmadeva, dont la titulature est identique à celle des rois du Malāyu connus par des inscriptions de 1286 à 1378 . A première vue, la date par laquelle débute l'inscription semble devoir être lue 11006, année du Lièvre, ce qui n'a pas de sens. M. Krom a suggéré à ma lecture deux corrections possibles (Hindoe-Javaansche Geschiedenis, p. 411, note 3). Il croit pouvoir discerner sous le second chiffre 1 un trait qui en ferait un 2 ou un 3 , et propose de prendre le second zéro comme la partie gauche d'un chiffre dont j'ai lu la partie droite comme le chiffre 6: la date serait donc 1204 ou 1304 çaka. D'un nouvel et minutieux examen de l'inscription, il résulte que la première correction n'est pas à retenir. Si quelque chose est sûr, c'est que les trois premiers chiffres doivent se lire 110. D'ailleurs, les années 1204 et 1304 çaka ne sont ni l'une ni l'autre des années du Lièvre. Par contre, la seconde correction de M. Krom est excellente : ce que j'ai pris pour deux chiffres n'en forme presque certainement

1) Hindoe-Javaansche Geschiedenis, pp. 110-111. 
qu'un. Est-ce 4 comme le suppose M. Krom? Pour en décider, étant donné que la lecture 110. est sûre, il n'y a qu'à se reporter au tableau des années cycliques et chercher celle qui fut une année du Lièvre entre 1100 et 1109 çaka. On trouve que c'est l'année 1105, ainsi que je l'avais d'ailleurs déjà indiqué (B. E. F. E.-O., XVIII, 6, p. 35, note 1).

L'inscription du Buddha de Grahi est donc de 1183 A.D., et si à cette date le nom d'un roi du Malāyu apparaît à Jaiya, c'est sans doute parce que le Malāyu avait déjà remplacé Çrīvijaya dans la suzeraineté sur les petits royaumes de la Péninsule.

Cette conclusion semble être en conflit avec le témoignage de Tchao Jou-koua qui décrit en 1225 le San-fo-ts'i comme un empire florissant, ayant sous sa dépendance tous ces royaumes de la Péninsule, y compris justement le Kia-lo-hi, c'est à dire Grahi. Mais n'oublions pas que les renseignements du Tchou fan tche ne sont pas basés sur une vision directe des choses. Tchao Jou-koua, directeur du commerce maritime dans le circuit du Fou-kien, n'avait pas voyagé lui-même dans les pays qu'il décrit, et son ouvrage est en partie copié textuellement sur le Ling wai tai ta de Tcheou K'iu-fei, en partie basé sur des renseignements oraux. La description générale du Sanfo-ts'i dans le Tchou fan tche de Tchao Jou-koua est inspirée du Ling wai tai ta et se rapporte par conséquent à la période antérieure à 1178. Par contre, la liste des états tributaires du San-fo-ts'i ne figure pas dans l'ouvrage de Tcheou K'iu-fei, et peut être postérieure à cette date. Or il y a dans cette liste un trait tout à fait frappant, c'est que parmi les dépendances du San-fo-ts'i figure Pa-lin-fong, c'est à dire Palembang!

Si le siège de l'empire qui groupait sous sa suzeraineté les différents royaumes de Sumatra et de la Péninsule Malaise était encore à Palembang, on ne comprend pas pourquoi Palembang figure parmi les dépendances de cet empire. Ni pour le Cambodge, ni pour Java, les listes d'états tributaires mentionnés par Tchao Jou-koua ne comportent pareille anomalie. On peut noter d'autre part que le Malāyu n'est pas cité parmi les dépendances du San-fo-ts'i. Ceci s'explique mal, si la liste se rapporte à une époque où le San-fo-ts'i, ayant sa capitale à Palembang, comptait certainement le Malāyu au nombre de ses tributaires; mais tout devient clair si cette liste est celle des dépendances du Malāyu, héritier du royaume de Palembang. Bref, on a l'impression que le chapitre du Tchou fan tche relatif au San-fo-ts'i a confondu sous ce titre des renseignements sur Çrivijaya 
antérieurs à 1178 (date du Ling zvai tai ta et aussi - ceci est important -- de la dernière ambassade du San-fo-ts'i à la cour de Chine mentionnée dans l'Histoire des Song), et des renseignements se rapportant à une époque où le centre de gravité de l'empire avait glissé vers le nord et se trouvait au Malāyu. Je ne crois donc pas qu'on puisse invoquer le témoignage de Tchao Jou-koua contre celui de l'inscription de Grahi qui nomme en 1183 un roi du Malāyu.

Que s'est-il passé entre 1178 et 1183 ? Je crois qu'il faut pour le moment se résoudre à l'ignorer. Il n'est peut-être pas nécessaire de chercher en-dehors de Sumatra, à Java par exemple la cause de la chute de Çrĩvijaya et de son remplacement par le Malāyu. L'ambition, jointe à la valeur personnelle d'un prince de Jambi, a pu suffire a produire une répétition des évènements qui se sont passés au Cambodge au milieu du VIme siècle, lorsque les Kambuja du Tchen-la se substituèrent à la dynastie qui régnait sur le Fou-nan. Mais, je le répète, il serait vain de chercher à tout expliquer. Contentons-nous d'avoir désormais une date assez précise pour la rentrée en scène du Malāyu.

Cet essor du Malāyu, dont le début remonte ainsi à la fin du XIIme siècle, c'est à dire un siècle plus tòt que ne le pense $M$. Krom, ne semble pas avoir eu sur la Péninsule Malaise de résultats bien durables. On sait de façon certaine que dès la fin du XIIIme siècle les Thai étaient maitres de tout le nord de la Péninsule jusqu'à Ligor, et rien n'autorise à supposer que Candrabhānu qui régnait dans cette région en 1230 ait été vassal du Malāyu. La liste des états tributaires de Sumatra donnée par Tchao Jou-koua, qui comprend le Tan-maling (Tāmbralinga), le Kia-lo-hi (Grahi) et d'autres pays de la Péninsule, est antérieure à 1225 . Or c'est peu de temps après cette date que se produisit en Indo-chine un évènement qui allait avoir un retentissement considérable et allait détruire l'équilibre des puissances : la libération des Thai de Sukhodya.

Un passage de l'histoire des Ming, souvent cité, dit qu'en 1295 les gens du Sien, c'est à dire les Thai de Sukhodaya, s'entretuaient depuis longtemps avec les Malāyu. Dès le début de son existence, le royaume de Sukhodaya semble avoir eu des vues sur la Péninsule Malaise. A propos de l'expédition à Ceylan du roi Çrīdharmarāja (= Candrabhānu) en 1256, la Jinakālamālinī raconte que le roi de Sukhodaya nommé Rocarāja (Brạ̣ Ruan des légendes siamoises), ayant eu envie de voir la mer, descendit le Ménam accompagné d'une nombreuse armée et arriva à Çrīdharmanagara (Ligor). Le roi Çrī- 
dharmarāja le reçut amicalement et lui parla des miracles qu'il avait entendu raconter au sujet d'une certaine statue de Ceylan. Le roi de Sukhodaya ayant conçu le désir de posséder cette statue, une mission fut envoyée à Ceylan .... ${ }^{\mathbf{1}}$ ). Candrabhānu trouva-til dans cette amitié du fondateur de la dynastie de Sukhodaya un moyen de se libérer de la suzeraineté du Malāyu, si tant est qu'il l'ait jamais effectivement subie? Il serait téméraire de l'affirmer. Ce qui est probable, c'est que la religion fut mêlée à la politique, ainsi que l'a judicieusement fait observer M. Krom (pp. 16-17).

La langue de l'inscription de Candrabhānu à Jaiya, bien que sanskrite trahit une forte influence pālie. Le passage de la Jinakālamālinī qui vient d'être cité, confirmé par certaines allusions du Mahāvạ̣sa signalées plus haut, dit clairement que la mission de Candrabhānu à Ceylan avait pour but l'obtention d'une image (le Mahāvaṃsa parle de reliques) du Buddha. L'état florissant du Hīnayāna à Ligor à la fin du XIIIme siècle est attestée par l'inscription de Rāma Gāmhèn roi de Sukhodaya qui fonda ,à l'ouest de la ville le monastère des Âraññika pour l’offrir au patriarche, au sage qui a étudié complètement les Trois Corbeilles, qui est plus docte que tous les autres moines du pays et qui est venu de Nagara Çrīdharmarāja.” Le Tāmbralinga, avant d'être définitivement incorporé par Rāma Gāṃhèn au royaume de Sukhodaya, forma peut-être avec celui-ci, en même temps qu'une coalition politique contre le Malāyu, une coalition religieuse hīnayāniste contre le Mahāyāna qui règnait en maître dans l'Archipel.

L'influence du Malāyu sur la Péninsule Malaise, qui est attestée aux environs de 1200 par l'inscription du Buddha de Grahi de 1183 et par la liste du Tchou fan tche antérieure à 1225, devait vers 1250 avoir perdu beaucoup de terrain au profit des Thai. Quel fut exactement son histoire à Sumatra même durant les deux premiers tiers du XIIIme siècle? Le silence que gardent nos sources sur l'histoire de l'île pendant cette période ne permet aucune conclusion. L'histoire de Sumatra ne recommence qu'en 1275, avec l'expédition de Kṛtanagara. M. Krom (p. 22) admet que c'est à la suite de cette expédition du roi de Singhasāri que ,l'état de Malāyu, vivant jusqu'alors sous la domination de Çrīvijaya, reconquit son indépendance grâce à la protection javanaise."

L'objet de la présente note est de montrer que la libération du

1) Cœdès, Documents .... pp. 98-99. 
Malāyu et la chute de Çrīvijaya remontent un siècle plus tôt, entre 1178 et 1183 , et que lorsque le Pararaton parle de l'expédition de Kṛtanagara contre le Malāyu, ce terme ne désigne pas nécessairement l'île de Sumatra en général, mais bel et bien le Malāyu ou royaume de Jambi, qui avait recueilli depuis un siècle la succession de Çrīvijaya ou royaume de Palembang.

\section{RÉSUMÉ CHRONOLOGIQUE.}

1178 Dernière ambassade du San-fo-ts'i (Çrīivijaya) à la cour de Chine citée par l'histoire des Song. - Description du San-fots'i dans le Ling wai tai ta.

Décadence de Çrīijaya. - Essor du Malāyu.

1183 Inscription de Trailokyarājamaulibhūṣaṇavarmadeva (roi du Malāyu à en juger par sa titulature) à Grahi (Jaiya).

1230 Inscription de Candrabhānu, roi du Tāmbralinga, à Jaiya.

1236 Première expédition de Candrabhānu à Ceylan.

1256 Visịte du roi de Sukhodaya à Ligor. - Seconde expédition de Candrabhānu à Ceylan avec l'aide des Pāṇḍya, qui finalement se retournent contre lui et l'anéantissent.

1275 Expédition de Kṛtanagara contre le Malāyu.

1281 Première ambassade du Malāyu à la la cour de Chine citée dans l'histoire des Yuen. 